\title{
On-demand routing protocols for cognitive radio ad hoc networks
}

\author{
Shelly Salim and Sangman Moh*
}

\begin{abstract}
Cognitive radio ad hoc networks (CRAHNs) are a class of cognitive radio networks. In recent years, they have gained popularity, and routing protocols have been proposed. Above all, the protocols based on on-demand routing are considered favorable in the literature. It is mainly because the accomplishments of ad hoc on-demand distance vector (AODV) routing and dynamic source routing (DSR) in mobile ad hoc networks have lead to a number of adaptations of both protocols to suit CRAHNs. In this paper, we review the on-demand routing protocols applicable for CRAHNs, which are based on AODV, DSR, and hybrid protocols. After explaining their basic principles, we qualitatively compare the protocols in terms of inherent characteristics and performance. This paper further addresses the pros and cons of routing protocols and discusses research challenges and open issues.
\end{abstract}

Keywords: CRAHN, Routing protocol, On-demand routing, AODV, DSR

\section{Review}

\section{Introduction}

Cognitive radios enable an adaptive approach in utilizing existing wireless spectrum. This approach introduces a different concept of physical layer operations and ultimately affects the whole upper layers. Despite the challenges, cognitive radios have become popular in the past few years. The main reasons are because they provide the ability for secondary users (SUs) to use and share the licensed spectrum bands opportunistically and support prioritization for the transmissions of licensed/primary users (PUs), simultaneously. Hence, cognitive radios are potential to increase the spectrum utilization. In order to use the licensed spectrum band, SUs must have at least one cognitive radio transceiver. With the cognitive radio transceivers, SUs search for vacant spectrum, called spectrum opportunity, by conducting spectrum sensing. Since PUs have the authority to use the licensed spectrum band, SUs must not interrupt the transmissions of PUs by performing reconfiguration of transmission parameters or moving to other vacant spectrum bands [1]. This creates dynamic use of spectrum bands, where the SUs are able to switch among different spectrums.

\footnotetext{
* Correspondence: smmoh@chosun.ac.kr

Department of Computer Engineering, Chosun University, 309 Pilmun-daero, Dong-gu, Gwangju 501-759, South Korea
}

The dynamic use of the spectrum bands creates adverse effects on network performance if the same communication protocols, which were developed considering a fixed frequency band, are applied. Therefore, new protocols should be designed appropriately to suit the cognitive radio network environment. Years ago, the studies in cognitive radio networks existed mainly in the physical and medium access control layers, such as spectrum sensing, spectrum sharing, and spectrum management. Nowadays, there are a number of works that propose routing protocols for cognitive radio networks. This might be triggered by the unique properties of cognitive radio networks that lead researchers to explore variety of methods to best suit cognitive radio networks: from adding some adaptations of existing routing protocols to creating completely new protocols.

In this paper, on-demand routing protocols applicable for cognitive radio ad hoc networks (CRAHNs) are classified and reviewed, and then challenges and open issues are discussed. The protocols are based on ad hoc ondemand distance vector (AODV), dynamic source routing (DSR), and hybrid on-demand routing protocols. Because of the challenging nature of CRAHNs, there are some unique issues encountered while designing a routing protocol. The comparison study shows that there are a number of challenges remaining in order to design a complete and feasible routing protocol for CRAHNs. 
In the following section, routing in CRAHNs is introduced and discussed. In the section 'Adaptation of ondemand routing to CRAHNs', the adaptation of on-demand routing protocols such as AODV, DSR, and hybrid protocols into CRAHNs are presented, and they are qualitatively compared. The next section discusses the challenges and open issues. Finally, this paper is concluded in the last section.

\section{Routing in CRAHNs}

CRAHNs are a class of cognitive radio networks without any fixed infrastructures or centralized entities [2]. Without any centralized body, SUs have to cooperate between themselves in an ad hoc manner to exchange information and obtain necessary knowledge, such as network topology and PUs' presence. Thus, the routing protocol for CRAHNs should satisfy the requirements of both cognitive radio networks (CRNs) and ad hoc networks, as shown in Figure 1. The main concern of cognitive radio communication by SUs is to avoid impeding the PUs' transmission. Therefore, it is necessary to select the route that satisfies both PU avoidance and SUs' endto-end communication in the routing protocol. In [3], a PU avoidance scheme is inserted in the route setup procedure. There are two significant times when the SUs should select appropriate channels for their communication: one time is at the beginning of the data transmission, and the other time is at the route repair occasion. The channel availability information is obtained from the spectrum sensing mechanism on the physical layer or spectrum occupancy database, if any [4]. Since the spectrum information is a crucial part of route determination, routing protocol should consider cross-layer approach with the physical layer, creating the so called 'spectrum awareness' [5].

The properties of ad hoc networks must also be covered in designing routing protocols for CRAHNs. Node mobility encourages spectrum utilization variation not only in time domain but also in space domain. For the

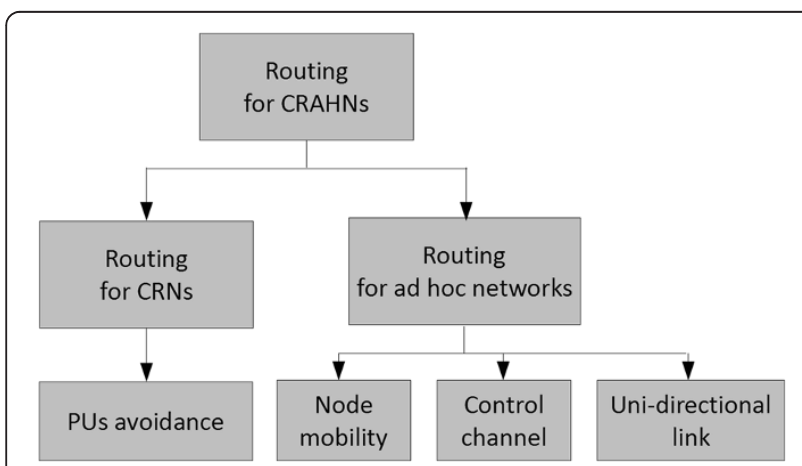

Figure 1 Routing protocol of CRAHNs should satisfy both cognitive CRN requirements and ad hoc network requirements. mobile SUs, the spectrum availability at the new location has to be immediately sensed. The adaptation of spectrum sensing result in real-time manner could be very challenging. Thus, efficient spectrum mobility procedure should be considered when designing the routing protocols for CRAHNs. Another characteristic of ad hoc networks is self-configuration. With the absence of a centralized body, all nodes should exchange network-related information in distributed manners via a control channel, so they could organize their data transmissions on the shared wireless medium with minimum transmission failure. In the cognitive radio network environment, the set of available spectrum bands might be different from node to node and might be scarce from time to time. Thus, dedicating some channels to be the common control channels is a questionable implementation. Besides having to solve the control channel issue, routing protocols designed for CRAHNs should not assume bi-directional links. Unidirectional links occur because different nodes might have diverse transmission ranges.

In addition to the abovementioned issues, some requirements of the traditional networking protocols should not be left behind in designing a routing protocol for CRAHNs. Such requirements include power efficiency, quality of service (QoS), and security. Especially in battery-powered mobile nodes, the power efficiency issue is particularly important. One effort to reduce the nodes' power consumption is to use on-demand routing mechanisms. On-demand routing protocols only establish a path when there is active communication taking place, thus reducing energy consumption. There are lengthy discussions on improving the performance of QoS and security that could be carried out by other layers. However, the routing protocol should at least support QoS as one of the considerations in selecting the available spectrum along the end-to-end route establishment.

\section{Adaptation of on-demand routing to CRAHNs}

In wireless ad hoc networks, AODV routing [6] and DSR [7] are the two representative routing protocols, and they are on-demand protocols. Some hybrid works of on-demand and proactive routing protocols are also favorable. In the AODV and DSR protocols, whenever data communication is needed, the source node would find a route to the destination; this is called route discovery. Route discovery starts with the source node broadcasting route request (RREQ) packets; then, the packets are forwarded by intermediate nodes or relaying nodes. The destination node or the intermediate node who knows the route to the destination node responds by unicasting a route reply (RREP) packet back to the source node. When the source receives RREP, the route is established, and data packet transmission may start. Besides route discovery, AODV and DSR also 
have route maintenance procedures involving route error (RERR) packets.

Two main characteristics of AODV are the setup of reverse/forward path pointers and destination/source sequence numbers. AODV uses sequence numbers as freshness indicator and loop-free guarantee [8]. Meanwhile, the main characteristics of DSR are the RREQ and RREP packets' format. As a source routing, the source node determines the complete sequence of hops to reach the destination. Thus, the RREQ and RREP packets contain all the intermediate nodes' addresses. AODV does not store any routing entries but the ongoing communication, while DSR maintains a route cache and uses the existing route entries, if available, until they are marked invalid [9]. In one of the performance comparison, AODV delivers almost $90 \%$ of the transmission packets, while DSR performs best with the minimum number of hops [10].

In mobile ad hoc networks (MANETs), on-demand routing requires less network resources and less energy consumption than proactive routing because the proactive routing has to broadcast control messages periodically and store all routing paths in the routing table even though there is no data to send. In general, proactive routing is more suitable for stable ad hoc networks, whereas on-demand routing is more suitable for dynamic ad hoc networks. Under the same environment and operation conditions, CRAHNs are more dynamic than MANETs due to the unexpected behavior of PUs. As a result, the on-demand routing is more suitable for CRAHNs rather than the proactive routing. AODV and DSR are the approved and standardized MANET routing protocols. They support the properties of ad hoc topology, self-organizing, self-configuring, and mobility well [11]. They also have route maintenance procedures that can be used to solve frequent connectivity changes. AODV is probably more suitable for cognitive wireless networks compared to DSR. One of the reasons is because DSR route discovery may lead to unpredictable length of control packets and data packets. Thus, DSR is not suitable for the intermittent connectivity environment of cognitive radio networks. Nevertheless, DSR adaptation for cognitive radio network routing protocol is one of the earliest works [12]. However, later, AODVbased cognitive routing protocols are proposed more than DSR-based ones.

As stated earlier, to apply the routing protocols of other wireless networks to cognitive radio networks is not feasible due to their poor performance in dynamic spectrum environment. Therefore, modifications are necessary in adapting AODV and DSR for cognitive radio routing. A common approach is by inserting spectrum-related information, such as spectrum opportunity (SOP), channel usage list, etc., of the SUs into the routing control packets. In the route discovery, this spectrum-related information is piggybacked on the control packets (RREQ, RREP, and RERR). For example, the source node may insert its spectrum-related information on RREQ packets. When the intermediate nodes forward the RREQ packets, they also include their own spectrum-related information. Then, usually the destination node gets to decide the spectrum band to be used for data transfer. It assigns the spectrum, encapsulates it in RREP, and sends it back to the source node. Likewise, intermediate nodes that receive RREP assign the spectrum bands (Figure 2). Hence, on RREQ and RREP forwarding, the packets are getting larger in size with agreement with the hop or distance between the source and destination. The routing protocols that adopt this method are as follows: spectrum-aware ondemand routing protocol (SORP) [13], delay-motivated on-demand routing protocol (DORP) [14], and weighted hop, spectrum awareness, and stability (WHAT) routing metric [15]. Some protocols assume that other information is also carried by RREQ and RREP, such as node states in multi-hop single-transceiver cognitive radio network routing protocol (MSCRP) [16]. However, most studies that add extra information to control packets did not evaluate the overhead. Some protocols propose solutions to specific problems such as the deafness problem, load balancing, and intermittent connectivity problem.

\section{Adaptation of AODV to CRAHNs}

A simple modification to adopt AODV into CRAHNs environment is by defining a new routing metric with 'spectrum awareness' property. Such protocols are SORP [13], DORP [14], and WHAT routing metric [15]. DORP is a continuation work of SORP, and both are early works that define the cumulative delay metric. WHAT routing metric considers path length, usage of licensed channel, and channel switching frequency.

The cumulative delay metric of SORP and DORP introduces switching delay, which is later referred by many other works. Switching delay is defined as the delay caused by switching among frequency bands. Another consideration is backoff delay that is caused by multi-

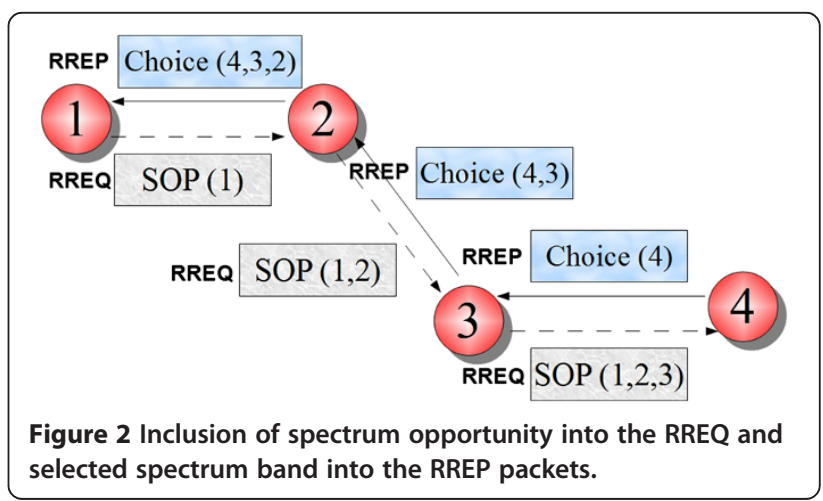


flow interference within a frequency band. Cumulative delay is the sum of switching delay and backoff delay. DORP adds queuing delay caused by the transmission of other flows on the node to the cumulative delay calculation. Since SORP and DORP focus on delays, they are suitable for delay-sensitive application. They result in fairly low cumulative delay and smooth cumulative delay change in handling intersecting flows. On the other hand, WHAT metric claims to be able to capture the overall quality of a path to have multiple considerations and to enhance the network throughput. However, WHAT metric requires a tuning parameter in determining the metric value and path selection. This static, predefined tuning parameter would reduce the cognitive learning capability.

Some routing protocols that provide spectrum awareness for route selection rather than employing new routing metric are connectivity-based routing [17], cognitive ad hoc on-demand distance vector (CAODV) [18], and dual diversity cognitive ad hoc routing protocol ( $\mathrm{D}^{2} \mathrm{CARP}$ ) [19]. The connectivity-based routing observes the SUs' network connectivity which is determined by the PUs' channel usage on the basis of graph theory and mathematical analysis. It finds all possible paths from source to destination by adopting the route discovery procedure of AODV. Then, the destination selects one best-quality path by considering the number of hops, PUs' activity, and channel switching along the paths. However, no performance comparison given is given in [17].

CAODV exploits spectrum diversity in the two ways of inter-route and intra-route spectrum diversity. Interroute spectrum diversity is a scheme to discover multiple routes but through the same channel, while intra-route spectrum diversity is a scheme to discover a single route but through multiple channels. CAODV introduces an additional control packet, which is primary user route error (PU-RERR) packet, to indicate an error caused by the detection of PUs' activity in the operating channel. PU-RERR packets are locally broadcasted on the channels affected by PUs' activity, and they provide a method to adapt to dynamic spectrum availability during data communications. However, performance comparison against any routing protocols is not given in [18]. $D^{2}$ CARP is a variation of AODV that combines path and spectrum diversity, that is, the SUs could switch among different paths and different channels. To support such capability, the route discovery process provides multi-path and multi-channel routes. $D^{2} C A R P$ improves CAODV in terms of packet delivery ratio, overhead, delay, and hop count. However, $\mathrm{D}^{2} \mathrm{CARP}$ requires RREP packets to be broadcasted back to the source, which requires a larger routing table and more resource consuming. Note that, in the original AODV, the RREP packet is unicasted to the source.
Routing protocols could also be designed to solve specific problems, such as the MSCRP trying to solve deafness problem [16] and local coordination-based routing focusing on load balancing [20]. MSCRP applies node states and deafness problem constraint. Each node must be in one of the three node states: single-channel state, switching state, and non-free state. When there is no flow across a node or there are flows only on the channel where the node stays, that node is at single-channel state. When the flows are distributed on different channels, the node is at switching state. A node is at non-free state when it is a neighbor of a switching state node. After applying those states, the deafness problem constraint is defined as two consecutive nodes in a flow cannot be simultaneously at switching state.

Local coordination-based routing is also a continuation work of SORP with a local coordination scheme on intersecting nodes to perform load balancing. In this protocol, once a node becomes an intersecting node to accommodate multiple data flows, the local coordination scheme is invoked. This scheme helps intersecting nodes to decide whether to perform flow accommodation or flow redirection based on workload evaluation (includes additional control packet exchanges). In Figure 3, node 1 is serving flow 2, and node 2 is serving flow 3 . When new flow 1 occurs, first it is established with node 1 and node 2 as intermediate nodes. These two intersecting nodes perform a local coordination scheme to find appropriate neighbors to redirect the flow. The results are as follows: node 1 redirects flow 1 to neighboring node 3 , and node 2 redirects flow 3 to neighboring node 4 .

However, further examined, local coordination routing has a contrasting approach with MSCRP. In local coordination routing, in order to balance the load between nodes, it is encouraged to redirect flows to neighboring nodes, thus creating switching nodes occurring side by

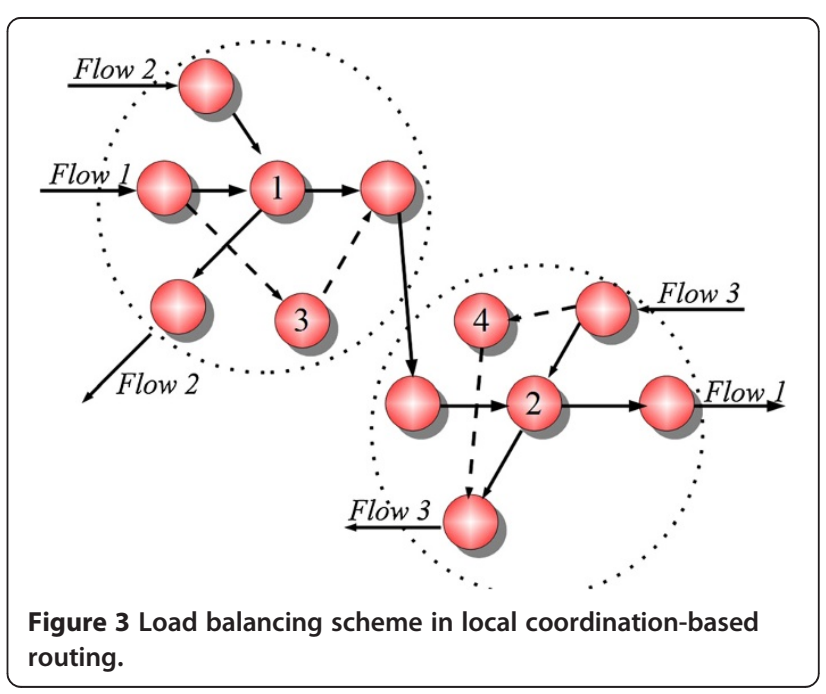


side. This violates the deafness problem constraint, that is, two consecutive nodes serving the same flow should not be switching nodes. It is difficult to decide which approach is better since MSCRP results in significant network throughput improvement and local coordination-based routing results in low cumulative delay.

Routing protocols that concentrate on path provision guarantee have also been proposed. Path provision could be maintained by either path reservation or backup path. Spectrum-aware routing protocol (SPEAR) has a channel reservation algorithm and aims to increase the network throughput [21], while the backup channel and cooperative channel switching (BCCCS) routing focuses on route maintenance by providing a backup channel [22]. SPEAR allows multiple paths to propagate to the destination node and limits the number of routes forwarded by each node by few additional parameters. After the destination node performs route selection using the traditional routing metric (maximum throughput and minimal hop count to break ties), embeds the channel assignment in RREP, and sends it to the source node, every node along the path then performs route setup and channel reservation by propagating explicit channel reservation messages to the neighbors within the interference impact area.

In BCCCS routing, each node saves a list of available channels and priorities and updates it periodically. BCCCS routing involves channel requests (CREQ), channel reply (CREP), and channel information (CINFO) control packets. CREQ works similarly to AODV's RREQ with the addition of cognitive radio identifier (CRID) of the source node and channel counters. CREP works similarly to AODV's RREP with the addition of CRID of both the source and destination nodes, link metric, and prioritized channel list. The CINFO messages are periodically sent to the neighboring nodes to refresh the list of available channels and priorities. For data transfer route, the destination node selects the least used channel by referring to the channel counters. When this route is broken, the involving nodes simply switch to a channel at the second priority on the available channel list and update the next backup channels.

Both SPEAR's channel reservation and BCCCS's backup channel objectives are to provide channel availability. Channel reservation attempts to guarantee an accessible channel from the beginning of the data communication, in other words, a proactive approach. A backup channel tries to solve immediate availability issue of the channel in the case of a broken path. On one hand, the proactive approach is favorable to obtain better spectrum management and sharing between the nodes and to minimize packet collision. On the other hand, in the cognitive radio environment, the available spectrum bands are dynamically varied over time and space, and the link reliability is relatively low. Thus, broken links might occur frequently. In those cases, the provision of a backup channel would result in immediate route recovery and avoids the repetition of the time- and resource-consuming route discovery scheme. Hence, providing a backup channel might be a better solution. The SPEAR performance, in its best case, could achieve more than 180\% throughput improvement over link-based routing, and in its worst case, it could achieve 35\% throughput improvement over link-based routing and flow-based routing. BCCCS routing is shown to be able to achieve almost $100 \%$ connectivity.

A quite advanced approach of AODV modification is traffic-aware routing protocol (TACR) that combines the traffic-aware routing and Q-learning algorithm [23]. In route discovery, intermediate nodes trigger the prediction model when RREQ is received. The prediction model formula includes the prediction time interval, standard normal random variable, and two constant controlling parameters. Moreover, TACR is able to perceive current traffic information by using a packet called cognitive packet. Traffic prediction and traffic perception are used as inputs to the route decision making process. TACR employs Q-learning technique in the route maintenance. Q-learning enables a network to self-configure, selfmanage, and self-adapt. When the $Q$-value reaches a certain threshold, it means that the quality of the path is better, and the route involving this path will increase the route lifetime. The simulation results of TACR are end-toend delay and packet loss rate reduction, as well as throughput improvement when traffic arrival rate is high.

\section{Adaptation of DSR and hybrid protocols to CRAHNs}

One of the earliest works on routing protocols for cognitive radio network is based on DSR; it is called collaborative routing. This work has been referred to many times as it explains the importance of collaborating route selection and spectrum management. However, a collaborative route needs a central entity, so it cannot be referred for CRAHNs. Another routing protocol that defines a new routing metric is cognitive routing metric with improving capacity (CRM-IC) [24]. CRM-IC aims to improve the network capacity by considering PUs' usage pattern and interference of the SUs. This routing metric improves the end-to-end throughput and transmission completion time.

Two similar works focus on intermittent connectivity problem; they are anti-intermittence source routing protocol (AiSorp) [25] and anti-intermittence routing [26]. AiSorp combines the forward routing discovery process of DSR and backward process of AODV. In its routing discovery, when the cognitive radio routing request reaches the destination node, the destination node stops transmitting and computes the corresponding path metric. The path metric considers the PUs' channel utilization, link weight, and two predefined parameters. For the route 
maintenance, when a path is invalid, the affected node does not immediately send a cognitive radio routing error (CR3ER) to the source node. Instead, the node waits for a period of time, called the routing holding state. Before the routing holding state timer expires, if the node can transmit data again, it then performs routing maintenance; else, it sends a CR3ER packet to the source node.

Likewise, anti-intermittence routing also focuses on the intermittence connectivity issues and contains some procedures similar to AiSorp. The difference in the antiintermittence routing is that it designs a routing metric to maximize route lifetime by taking into account the probability and the average time that the channel is free from PUs, intra-flow interference, and three weighing parameters. Also, in the route maintenance, when a channel is invalid, the node will send CR3ER to the destination node instead of to the source node. The destination will collect all link information again. If the route path still exists, the destination node carries out the channel selection and selects a new channel for the whole path; otherwise, it performs re-routing.

Another hybrid routing protocol is the spectrum-tree -based on-demand routing protocol (STOD-RP) [27]. It combines tree-based proactive routing and on-demand route discovery from AODV. STOD-RP introduces a new metric, called airtime cost, to evaluate the resource consumption of a link. Airtime cost takes into account the channel access overhead, protocol overhead, and packet size (which is constant for specific access technology) to formulate link rate and packet error rate. Then, the available time duration of the spectrum band is included to evaluate the link stability. Finally, the cost of an end-to-end route, or the cumulative cognitive route cost, is the total airtime cost added with the total switch delay referred from SORP.

In STOD-RP, the SUs form a tree in each available spectrum band, called spectrum-tree. Each spectrum-tree has one root. Some nodes may belong to multiple spectrum-trees, called overlapping nodes or gateway nodes. The overlapping nodes are equipped with multiple spectrum-agile radios and simultaneously work in multiple spectrum-trees. Each node has its unique CRID in one spectrum-tree. Consequently, the overlapping nodes that work in multiple spectrum bands have multiple CRIDs. In this way, CRID indicates the proactive route to the root node. The STOD-RP routing is classified into intra-spectrum routing and inter-spectrum routing. The route discovery uses a spectrum route request (SRREQ) and a spectrum route reply (SRREP). SRREQ extends AODV's RREQ with the fields [CRIDS, CRIDD, metric, intra/inter], and SRREP extends AODV's RREP with the fields [CRIDS, CRIDD, intra/inter]. [CRIDS] and [CRIDD] are the CRIDs of the source node and destination node, respectively. [metric] is the cumulative cognitive route cost, and [intra/inter] indicates whether or not the destination node is in the same spectrum-tree as the source node. In the simulation results, when the number of gateway nodes increases, STOD-RP reduces the average endto-end delay, and as the number of channel increases, it reduces the control overhead. The routing protocols reviewed are summarized in Table 1.

\section{Challenges and open issues Novel routing metrics}

Working in a CRN environment, a routing protocol has another extra task to ensure that the communication between SUs does not disturb the PUs' communication. In order to do so, the routing mechanism should obtain the spectrum occupancy information on the physical layer and include the spectrum/channel assignment in the path selection so that the nodes would have to follow the assigned path and spectrum. A simple yet feasible approach is to adopt spectrum occupancy-related routing metric, such as PUs' existence probability, PUs' spectrum occupancy period, and interference level.

There are some works that introduce routing metrics for cognitive radio networks [28,29]. In [28], several routing metrics are introduced and combined. The routing metrics attempt to capture the end-to-end performance of SUs and the interference to PUs. A metric called OPERA is introduced for CRAHNs in [29], which is claimed to achieve optimality and accuracy. It focuses on actual end-to-end delay, and thus, it is suitable for delay-sensitive applications. These routing metrics try to involve the expected consequence of the SUs' route establishment to the PUs' communications quality.

Some studies referred in this paper define a routing metric that considers the PUs' spectrum occupancy period. However, that approach is only appropriate when the PUs are occupying a certain spectrum in a regular manner, and it would likely be unsuitable when the PUs' behavior is unpredictable (unless a strong learning module is included to cope with the PUs' behavior). For a more realistic routing metric, one possible candidate is the metric based on interference with PUs. By monitoring the interference level, SUs could get a full picture of the PUs' occupancy and ongoing transmission power level on the spectrum band, thus avoiding those spectrums in their communication. One of the interference-based metric is the Federal Communications Commission (FCC) interference temperature [30]. However, there is one general drawback of the FCC interference temperature model, that is, the assumption that the SUs are aware of the PUs' interference temperature limits. The interference temperature limit information dissemination is considered a fundamental issue, and the FCC's interference temperature model was once dropped due to its real implementation issue [31]. Despite the 
Table 1 Comparison of routing protocols for CRAHNs

\begin{tabular}{|c|c|c|}
\hline Routing protocols & Advantages & Disadvantages \\
\hline \multirow[t]{2}{*}{ SORP [13] and DORP [14] } & - Suitable for delay-sensitive application & - Does not include PU avoidance \\
\hline & - Simulation result: lower cumulative delay & \\
\hline \multirow[t]{2}{*}{ WHAT routing [15] } & - Multiple consideration on metric calculation & - Uses manually defined parameter \\
\hline & - Simulation result: enhances throughput & \\
\hline \multirow[t]{2}{*}{ Connectivity-based routing [17] } & $\begin{array}{l}\text { - Observes connectivity of SUs' network by } \\
\text { considering PUs' activity }\end{array}$ & $\begin{array}{l}\text { - Discovers all possible paths during route discovery, } \\
\text { which might result in high resource consumption }\end{array}$ \\
\hline & $\begin{array}{l}\text { - Includes PUs' activity and channel switching } \\
\text { in the routing metric }\end{array}$ & \\
\hline CAODV [18] & $\begin{array}{l}\text { - Considers spectrum diversity, which provides } \\
\text { adaptability to PUs' activity }\end{array}$ & $\begin{array}{l}\text { - Discovers multi-path or multi-channel routes, which } \\
\text { might result in high resource consumption }\end{array}$ \\
\hline$D^{2}$ CARP [19] & $\begin{array}{l}\text { - Considers spectrum diversity, which provides } \\
\text { adaptability to PUs' activity }\end{array}$ & $\begin{array}{l}\text { - Discovers multi-path and multi-channel routes and } \\
\text { broadcasts RREP packets, which might result in high } \\
\text { resource consumption }\end{array}$ \\
\hline \multirow[t]{2}{*}{ MSCRP [16] } & - Solves the deafness problem & - Adds node networking tasks \\
\hline & - Simulation result: significantly improves throughput & \\
\hline \multirow[t]{2}{*}{ Local coordination-based routing [20] } & - Provides load balancing & - Adds control packet exchanges \\
\hline & - Simulation result: incurs fairly lower cumulative delay & \\
\hline \multirow[t]{2}{*}{ SPEAR [21] } & - Provides channel reservation & - Uses traditional routing metric \\
\hline & $\begin{array}{l}\text { - Simulation result: achieves significant throughput } \\
\text { improvement }\end{array}$ & - Uses manually defined parameter \\
\hline \multirow[t]{2}{*}{ BCCCS [22] } & - Provides backup channel & - Adds list and table keeping \\
\hline & - Simulation result: shows almost $100 \%$ connectivity & \\
\hline \multirow[t]{2}{*}{ TACR [23] } & - Includes learning capability & - Uses manually defined parameter \\
\hline & $\begin{array}{l}\text { - Simulation result: reduces end-to-end delay and } \\
\text { packet loss rate }\end{array}$ & - Adds complexity \\
\hline \multirow[t]{2}{*}{ CRM-IC [24] } & - Maximum capacity route selection & - Uses single routing metric \\
\hline & $\begin{array}{l}\text { - Simulation result: larger end-to-end throughput and } \\
\text { better transmission completion time }\end{array}$ & \\
\hline \multirow[t]{2}{*}{ AiSorp [25] } & - Defines route maintenance procedure modification & - Uses manually defined parameters \\
\hline & - Simulation result: longer routing lifetime & \\
\hline \multirow[t]{2}{*}{ Anti-intermittence routing [26] } & - Defines route maintenance procedure modification & - Uses manually defined parameters \\
\hline & - Simulation result: longer routing lifetime & \\
\hline \multirow[t]{2}{*}{ STOD-RP [27] } & - Provides proactive route via tree-based routing & \multirow{2}{*}{$\begin{array}{l}\text { - Lacks analysis of gateway node activity (e.g., energy } \\
\text { consumption) }\end{array}$} \\
\hline & $\begin{array}{l}\text { - Simulation result: reduces end-to-end delay and } \\
\text { control overhead }\end{array}$ & \\
\hline
\end{tabular}

implementation issue, there is a possibility that the FCC's interference temperature model would be revived since there are efforts to model the interference temperature constraint for CRNs as in [32]. Therefore, the interferencebased routing metric is seen to be favorable and promising for implementation in CRAHNs.

\section{Network resource consumption}

In CRNs, SUs access and communicate using the vacant spectrum band opportunistically. This condition sets a limitation on network resources. In a condition where the penetration of PUs' activity is high, SUs might have only a few vacant channels for their packet transmissions. Thus, they should manage their packet transmissions efficiently, possibly by reducing the number of control packet transmissions and focusing on data packet transmissions. Ondemand protocols do not require periodic control packet exchanges, but they broadcast and unicast control packets in the route discovery and route maintenance procedures for active routes. In other words, by eliminating periodic control packet exchanges, on-demand protocols reduce the number of packet transmissions required by SUs. Some routing protocols based on on-demand routing in CRAHNs, such as the connectivity-based routing [17], CAODV [18], and $\mathrm{D}^{2}$ CARP [19], add the control packet transmissions. They discover multiple paths during the 
route discovery. Furthermore, CAODV adds probe packets and additional control packet PU-RERR, whereas $D^{2}$ CARP broadcasts RREP packets back to the source. The effectiveness of adding control packet transmissions against the channel availability should be considered.

\section{Network heterogeneity}

Heterogeneity in CRAHNs is not only caused by the dynamics of spectrum availability but also by the reconfigurability of SUs. The available spectrum for SUs may be different from node to node. Most of the routing protocols address the spectrum diversity by jointly selecting path and channel and by considering channel switching events. ARDC [33] adopts graph modeling to adapt to dynamic changes in the network topology efficiently. However, ARDC failed to consider the effect of SUs' reconfigurability. When the operating channel of a SU is rendered unavailable due to the detection of PUs' activity, the routing protocol would redirect the communication through another vacant channel, without considering reconfigurability. There is a wide range of reconfigurability supported by SUs with their cognitive radio devices, that is, transmission power, modulation scheme, coding rate, etc. can be reconfigured. These reconfigurability options can be combined with the routing decision. For example, by adjusting the transmission power and modulation scheme, the SUs might change the communication technique from overlay to underlay, without switching to another channel. By considering the reconfigurability of SUs, various options for routing decision are possible.

\section{Energy conservation}

When considering a network with mobile node deployment, energy conservation is inevitable since mobile nodes are often battery-powered. In CRAHNs, energy conservation of nodes is crucial because they have some extra particular tasks compared to noncognitive radio network. In the previous discussion, it has been pointed out that routing in CRAHNs requires further complexities; those are novel routing metric, learning module, and route maintenance. However, above all, the routing protocol should be as simple as possible to conserve energy. The works referred to in this paper fail to pay attention to this issue, except AiSorp and anti-intermittence routing, both of which increase the routing lifetime, and local coordination-based routing that focuses on load balancing. However, local coordination-based routing requires additional control packet exchanges for its workload evaluation, while it does not study the energy consumption of doing so. Nevertheless, for all referred works, since the routing protocols are on-demand based, they support simple energy conservation by setting up the route only when it is needed.
To preserve network lifetime, analyzing the energy consumption of additional tasks given to the nodes by the routing protocol is encouraged. For example, inserting the spectrum-related information into the routing control packets (RREQ, RREP, and RERR) would increase the packet size accordingly to the distance/hop traveled. The nodes have to perform extra task to attach the spectrumrelated information as well as to send longer packets. Those additional activities are energy consuming. Therefore, node-burdening tasks should be avoided if the performance is not fairly improved. Trade-off between energy consumption and additional node tasks (MSCRP's deafness problem constraint, BCCCS' list and table keeping, and STOD-RP's gateway node power usage) should be evaluated.

\section{Quality of service}

An addition to the complexities of CRAHN routing protocol design is to create QoS supporting routing protocols. The applications in CRAHNs might vary as in other networks, and they need different QoS. The basic categories of services are bandwidth, latency, jitter, and packet loss [34]. The applications might only need one of the services or a combination of them. However, in the referred works, we could not find any routing protocol with QoS support. Even though providing QoS requires additional computation, it is advantageous for SUs, especially when there are various kinds of application traffic with different service requests. By defining QoS, spectrum management becomes more efficient. For example, given a set of available spectrum band, there are two kinds of applications run by a SU: data transfer and voice communication. The routing protocol with QoS support could recognize the application service demands and would choose the path with the lowest loss for data transfer, lowest end-to-end delay, and lowest jitter for voice communication. Without QoS support, the routing protocol would assign the path and spectrum based solely on its routing metric and might fail to satisfy the application requirements.

\section{Conclusions}

This survey paper presents a number of on-demand routing protocols for cognitive radio ad hoc networks. They are classified based on the underlying on-demand protocols: AODV, DSR, and hybrid on-demand protocols. It turns out that routing protocols that modify AODV are the most popular ones. AODV is probably more suitable for cognitive wireless networks compared to DSR. One of the reasons is because DSR route discovery may lead to unpredictable packet length, which is not suitable for intermittent connectivity environment of cognitive radio networks.

Looking at existing works and discussions on routing protocol design for CRAHNs, an appropriate routing 
protocol could be derived. Firstly, we recommend an adaptation of on-demand routing since its performance has been proven to suit mobile ad hoc networks, and it has the preferred properties suitable for CRAHNs. Then, a novel routing metric should be defined to include spectrum-related information in the routing mechanisms. In this way, the path selection consists of not only the selected path but also of the assigned spectrum. One promising candidate is a metric that is based on interference level with PUs since it guarantees PU avoidance. The routing protocol should consider the network resource consumption by examining both the necessity of multiple paths and the addition of control packet exchanges. Moreover, the routing protocol should be aware of network heterogeneity by considering the reconfigurability of SUs as one of the routing options. To preserve energy, the routing protocol should be as simple yet effective as possible. The trade-off between energy consumption and additional node tasks should be evaluated, especially when the extra tasks are oriented to a single objective and not overall network performance improvement. Finally, the routing protocol should consider QoS support, which would be beneficial to SUs.

\section{Competing interests}

The authors declare that they have no competing interests.

\section{Acknowledgments}

The authors wish to thank the editor and anonymous reviewers for their helpful comments on this paper. This work was supported in part by the research fund from Chosun University, 2013.

Received: 5 October 2012 Accepted: 26 March 2013 Published: 16 April 2013

\section{References}

1. IF Akyildiz, WY Lee, MC Vuran, M Shantidev, NeXt generation/dynamic spectrum access/cognitive radio wireless networks: a survey. Comput. Networks 50(13), 2127-2159 (2006)

2. IF Akyildiz, WY Lee, KR Chowdhury, CRAHNs: cognitive radio ad hoc networks. Ad Hoc Networks 7, 810-836 (2009)

3. KR Chowdhury, MD Felice, SEARCH: a routing protocol for mobile cognitive radio ad-hoc networks. Comput. Comm. 32, 1983-1997 (2009)

4. G Ko, AA Franklin, SJ You, JS Pak, MS Song, CJ Kim, Channel management in IEEE 802.22 WRAN systems. IEEE Comm. Mag 48(9), 88-94 (2010)

5. M Cesana, F Cuomo, E Ekici, Routing in cognitive radio networks: challenges and solutions. Ad Hoc Networks 9(3), 228-248 (2011)

6. C Perkins, E Belding-Royer, Ad hoc on-demand distance vector (AODV) routing. (IETF RFC 3561, 2003). http://www.ietf.org/rfc/rfc3561.txt. Accessed 24 January 2011

7. D Johnson, Y Hu, D Maltz, The dynamic source routing protocol (DSR) for mobile ad hoc networks for Ipv4. (IETF RFC 4728, 2007). http://www.ietf.org/ rfc/rfc4728.txt. Accessed 24 January 2011

8. C Perkins, E Royer, Ad-hoc on-demand distance vector routing, in Proceedings of the 2nd IEEE Workshop on Mobile Computing Systems and Applications (New Orleans, 25-26 February 1999)

9. DB Johnson, DA Maltz, Dynamic source routing in ad hoc wireless networks, in Mobile Computing, ed. by T Imielinski, H Korth (Kluwer, Netherlands, 1996), pp. 153-179

10. S Mittal, P Kaur, Performance comparison of AODV, DSR and ZRP routing protocols in MANETs, in Proceedings of the International Conference on Advances in Computing, Control, and Telecommunication Technologies (Trivandrum, 28-29 December 2009), pp. 28-29
11. ASK Pathan, CS Hong, Routing in mobile ad hoc networks, in Guide to Wireless Ad Hoc Networks (Springer, London, 2009), pp. 59-92

12. Q Wang, $\mathrm{H}$ Zheng, Route and spectrum selection in dynamic spectrum networks, in Proceedings of the 3rd IEEE Consumer Communications and Networking Conference (Las Vegas, 8-10 January 2006)

13. G Cheng, W Liu, Y Li, W Cheng, Spectrum aware on-demand routing in cognitive radio networks, in Proceedings of the 2nd IEEE International Symposium on New Frontiers in Dynamic Spectrum Access Networks (Dublin, 17-20 April 2007)

14. G Cheng, W Liu, Y Li, W Cheng, Joint on-demand routing and spectrum assignment in cognitive radio networks, in Proceedings of the IEEE International Conference on Communications (Glasgow, 24-28 June 2007)

15. J Chen, H Li, J Wu, WHAT: a novel routing metric for multi-hop cognitive wireless networks, in Proceedings of the 19th Annual Wireless and Optical Communications Conference (Shanghai, 14-15 May 2010)

16. H Ma, L Zheng, X Ma, Y Luo, Spectrum aware routing for multi-hop cognitive radio networks with a single transceiver, in Proceedings of the 3rd International Conference on Cognitive Radio Oriented Wireless Networks and Communications (Singapore, 15-17 May 2008)

17. A Abbagnale, F Cuomo, Gymkhana: a connectivity-based routing scheme for cognitive radio ad hoc networks, in Proceedings of the IEEE Conference on Computer Communications (INFOCOM) Workshops (San Diego, 15-19 March 2010)

18. AS Cacciapuoti, M Caleffi, L Paura, Reactive routing for mobile cognitive radio ad hoc networks. Ad Hoc Networks 10(5), 803-815 (2012)

19. MA Rahman, M Caleffi, L Paura, Joint path and spectrum diversity in cognitive radio ad-hoc networks. EURASIP Journal on Wireless Communications and Networking 2012, 235 (2012). doi:10.1186/1687-14992012-235

20. Z Yang, G Cheng, W Liu, W Yuan, W Cheng, Local coordination based routing and spectrum assignment in multi-hop cognitive radio networks. Mobile Network. Appl. 13, 67-81 (2008)

21. A Sampath, L Yang, L Cao, H Zheng, BY Zhao, High throughput spectrumaware routing for cognitive radio based ad-hoc networks, in Proceedings of the 3rd International Conference on Cognitive Radio Oriented Wireless Networks and Communications (Singapore, May, 2008)

22. M Zeeshan, MF Manzoor, J Qadir, Backup channel and cooperative channe switching on-demand routing protocol for multi-hop cognitive radio ad hoc networks (BCCCS), in Proceedings of the 6th International Conference on Emerging Technologies (Islamabad, 18-19 October 2010)

23. $Y X u, M$ Sheng, $Y$ Zhang, Traffic-aware routing protocol for cognitive network, in Proceedings of the IEEE 72nd Vehicular Technology Conference (Ottawa, 6-9 Sept. 2010)

24. Z Zhong, T Wei, Cognitive routing metric with improving capacity (CRM-IC) for heterogeneous ad hoc network, in Proceedings of the International Conference on Information Networking and Automation (Kunming, 18-19 October 2010)

25. L Gong, S Deng, W Tang, S Li, Anti-intermittence source routing protocol in distributed cognitive radio network, in Proceedings of the 4th International Conference on Wireless Communications, Networking and Mobile Computing (Dalian, 12-14 October 2008)

26. H He, L Gong, W Tang, J Wang, S Li, Spectrum-aware anti-intermittence routing in distributed cognitive radio network, in Proceedings of the International Conference on Communications, Circuits and Systems (Milpitas, 23-25 July 2009)

27. G Zhu, IF Akyildiz, G Kuo, STOD-RP, A spectrum-tree based on-demand routing protocol for multi-hop cognitive radio networks, in Proceedings of the IEEE Global Telecommunications Conference (, New Orleans, 30 November-4 December 2008)

28. G Lei, W Wang, T Peng, W Wang, Routing metrics in cognitive radio networks, in Proceedings of the 4th IEEE International Conference on Circuits and Systems for Communications (Shanghai, 26-28 May 2008)

29. M Caleffi, IF Akyildiz, L Paura, OPERA: optimal routing metric for cognitive radio ad hoc networks. IEEE Trans. Wireless. Comm. 11(8), 2884-2894 (2012)

30. PJ Kolodzy, Interference temperature: a metric for dynamic spectrum utilization. Int. J. Netw. Manag. 16(2), 103-113 (2006)

31. MD Gallagher, KD Smith, FR Wentland, M Settle, NTIA comments on the establishment of an interference temperature metric to quantify and manage interference and to expand available unlicensed operation in certain frequency bands. (ET Docket No. 03-237, 2004). http://www.ntia.doc.gov/fcc-filing/2004/ ntia-comments-establishment-interference-temperature-metric-quantify-andmanage-inte. Accessed 25 November 2011 
32. J Bater, H Tan, KN Brown, L Doyle, Modelling interference temperature constraints for spectrum access in cognitive radio networks, in Proceedings of the IEEE International Conference on Communications (Glasgow, 24-28 June 2007)

33. J Li, Y Zhou, L Lamont, Routing schemes for cognitive radio mobile ad hoc networks, in Cognitive Radio Mobile Ad Hoc Networks, ed. by RF Yu (Springer, New York, 2011), pp. 227-248

34. W Weiss, QoS with differentiated services. Bell Labs Technical Journal 3(4), 48-62 (1998)

doi:10.1186/1687-1499-2013-102

Cite this article as: Salim and Moh: On-demand routing protocols for cognitive radio ad hoc networks. EURASIP Journal on Wireless

Communications and Networking 2013 2013:102.

Submit your manuscript to a SpringerOpen ${ }^{\circ}$ journal and benefit from:

- Convenient online submission

- Rigorous peer review

- Immediate publication on acceptance

- Open access: articles freely available online

- High visibility within the field

- Retaining the copyright to your article

Submit your next manuscript at $\gg$ springeropen.com 